\title{
Comparação do valor nutricional de dez cardápios segundo quatro programas computacionais
}

\author{
Comparison of nutritional values of ten menus \\ based on four computer programs
}

Francilene Gracieli Kunradi VIEIRA'

Patrícia Faria Di PIETRO'

Larissa da Cunha FEIO'

Maria Alice Altenburg de ASSIS

Marco Aurélio PERES²

Francisco de Assis Guedes de VASCONCELOS ${ }^{1}$

\section{RE S U M O}

\section{Objetivo}

Comparar os valores de energia e os nutrientes de 10 cardápios à luz de 4 programas computacionais de análise de consumo alimentar utilizados no Brasil.

\section{Métodos}

Foram selecionados os programas NutWin, DietWin, DietPro e Virtual Nutri. Foram analisados o valor energético e 11 nutrientes de 101 alimentos, que compunham 10 cardápios. Para testar diferenças do valor nutricional dos 10 cardápios entre os programas, foram utilizados os testes de Kruskal-Wallis e Mann-Whitney.

\section{Resultados}

Os programas variaram em relação ao número de alimentos (934 a 3 815), de nutrientes (28 a 144) e das principais fontes utilizadas. Não foram encontradas diferenças significantes dos valores de energia e macronutrientes entre os 4 programas. Em relação aos demais, o programa Diet Pro apresentou menores valores de gordura saturada, gordura monoinsaturada, gordura poliinsaturada, de ferro e sódio; o programa Virtual Nutri apresentou menores valores de fibra.

\section{Conclusão}

Os resultados deste estudo não permitem inferências quanto à qualidade das bases de dados e nem indicam a superioridade de quaisquer desses programas que justifique sua utilização preferencial na prática do

\footnotetext{
1 Universidade Federal de Santa Catarina, Centro de Ciências da Saúde, Programa de Pós-Graduação em Nutrição. Caixa Postal 476, Campus Universitário, Trindade, 88040-900, Florianópolis, SC, Brasil. Correspondência para/Correspondence to: P.F.D. PIETRO. E-mail: <fariadipietro@gmail.com>.

2 Universidade Federal de Santa Catarina, Centro de Ciências da Saúde, Programa de Pós-Graduação em Saúde Pública. Florianópolis, SC, Brasil.
} 
profissional de nutrição ou do pesquisador do consumo alimentar. Os resultados encontrados apontam para a necessidade de revisão do desenho dos programas computacionais e de suas bases de dados de nutrientes - para o planejamento e a análise do consumo alimentar.

Termos de indexação: Alimentos. Bases de dados. Inquéritos alimentares. Nutrição. Nutrientes. Software.

\section{A B S T R A C T}

\section{Objective}

To compare values of energy and nutrients of 10 menus based on four softwares used for food consumption analysis in Brazil.

\section{Methods}

The selected softwares were NutWin, DietWin, DietPro and Virtual Nutri. Values of energy and eleven nutrients from 101 foods were analyzed, divided into 10 menus with established portion sizes. Differences in nutritional values obtained for the ten menus were tested using Kruskal-Wallis and Mann-Whitney tests.

\section{Results}

The programs varied in relation to number of foods $(934-3,815)$, number of nutrients $(28-144)$ and main sources used. Comparing to the other three softwares, significantly lower values of saturated fat, monounsaturated fat, polyunsaturated fat, iron and sodium were found in the DietPro software; significantly lower values of fiber were found in the Virtual Nutri software.

\section{Conclusion}

The results of this study do not allow inferences related to the quality of the databases or indicate superiority of any one of the programs, to be used in practice by the nutrition professional or in research about food consumption. Results suggest the need of design revision and the nutrient database of softwares used in planning and analyzing food consumption.

Indexing terms: Food. Databases. Diet surveys. Nutrition. Nutrients. Software.

\section{N T R O D U Ç Ã O}

A análise do consumo alimentar humano é uma tarefa complexa que exige precisão na coleta de dados e na conversão dessas informações em quantidades de energia e nutrientes ${ }^{1}$. Estão disponíveis no mercado diversos programas computacionais que se propõem a auxiliar os profissionais na realização de orientações nutricionais, no cálculo e no planejamento de cardápios, na educação nutricional, nos cálculos e nas análises de parâmetros utilizados na avaliação nutricional (antropométrico, consumo alimentar e análise de nutrientes) e na formulação de dietas especiais ${ }^{2,3}$.

No entanto, a escolha do melhor programa tem sido uma decisão difícil em função do número e das características operacionais disponíveis, bem como das diferenças encontradas nas bases de dados de nutrientes ${ }^{3}$ utilizadas pelos respectivos programas.

O ponto crucial para a escolha de um programa de análise de consumo alimentar é a qualidade da base de dados de alimentos e nutrientes ${ }^{2,4}$, que pode variar dependendo do número de alimentos e nutrientes incluídos, das fontes utilizadas na elaboração da base de dados (tabelas de composição de alimentos, rótulos de alimentos comerciais ou fontes oficiais) e da maneira como o programa apresenta os valores ausentes dos nutrientes ${ }^{4}$. Outras características importantes desses programas dizem respeito à sua capacidade de comparar os resultados com padrões dietéticos recomendados, de armazenar arquivos ou pastas, de imprimir relatórios e de exportar os dados para outros aplicativos ${ }^{3,4}$.

Vários estudos internacionais compararam características operacionais e bases de dados de 
nutrientes e de alimentos entre diferentes programas ${ }^{3,5-11}$. Entretanto, são escassos os estudos comparativos entre os programas de análise de consumo alimentar disponíveis no mercado nacional12. Assim, este estudo tem por objetivo comparar os valores de energia e os nutrientes de 10 cardápios, calculados com a utilização de 4 programas computacionais de análise de consumo alimentar utilizados no Brasil.

\section{MÉ TO D OS}

Selecionaram-se os programas NutWin 2.5 ${ }^{13}$, DietWin Clínico 3.0 $0^{14}$, DietPro $4.0^{15}$ e Virtual Nutri $1.0^{16}$, por serem freqüentemente utilizados em pesquisas brasileiras sobre o consumo alimentar ${ }^{17-19}$, tendo em vista a experiência dos pesquisadores com tais programas, sua disponibilidade ao usuário particular ou seu fácil acesso pela rede mundial de computadores (web).

Primeiramente os programas foram caracterizados segundo: a) número de alimentos e nutrientes que compõem a base de dados; b) as principais fontes de dados; c) a capacidade do programa em exportar os dados para outros aplicativos; d) a habilidade em comparar os resultados com padrões dietéticos recomendados; e) a capacidade de armazenar arquivos ou pastas; f) a capacidade de imprimir relatórios; g) a disponibilidade do tamanho da porção dos alimentos em medidas caseiras e em unidades de peso do sistema métrico decimal; h) a disponibilidade de informação sobre tipos de refeições e horários na elaboração de planos alimentares ou na avaliação do consumo alimentar; i) o modo de apresentação dos valores ausentes de nutrientes (espaço em branco, valor zero ou não citados como parte da sua composição nutricional); e j) o fato de considerar ou não o álcool na distribuição percentual da quantidade de energia total.

Para comparar as bases de dados de nutrientes entre os 4 programas foram elaborados, por um nutricionista, 10 cardápios, baseados na dieta-padrão de 2 200Kcal, estabelecida pelo guia da Pirâmide Alimentar Adaptada para a Popu- lação Brasileira, efetuando-se a substituição dos alimentos por porções equivalentes das listas de substituição ${ }^{20}$. Foram utilizados 101 alimentos básicos para a construção desses cardápios (Tabela 1). O tamanho das porções e as medidas caseiras foram convertidos em pesos (gramas) e volumes (mililitros) por uma única fonte ${ }^{20}$, permitindo-se, assim, uma avaliação, diretamente nos programas, do conteúdo dos nutrientes e da energia fornecidos pelos alimentos e preparações. Os alimentos que não estavam disponíveis em um dos programas foram substituídos por outros do mesmo grupo alimentar. Em cada programa os dados dos 10 cardápios foram digitados em dupla entrada.

Para verificar a variação entre as bases de dados dos programas foram examinados os conteúdos de energia e 11 nutrientes básicos: proteína, carboidrato, gordura total, gordura saturada, gordura monoinsaturada, gordura poliinsaturada, colesterol, fibra, ferro, cálcio e sódio. A escolha desses nutrientes foi efetuada em função da presença obrigatória da maioria deles na rotulagem nutricional exigida pela Agência Nacional de Vigilância Sanitária e Alimentar (ANVISA) ${ }^{21}$. Além dos nutrientes exigidos pela ANVISA, foram realizadas análises dos conteúdos de gordura monoinsaturada, gordura poliinsaturada e colesterol, em razão do papel desses nutrientes no desenvolvimento de doenças crônicas não transmissíveis ${ }^{22}$. Os minerais ferro e cálcio, por sua vez, foram avaliados em função dos seus benefícios à saúde, tais como prevenção da anemia e osteoporose ${ }^{22}$.

Para as análises estatísticas utilizou-se o teste de Kruskall-Wallis para as comparações dos valores de energia, de macronutrientes e de micronutrientes entre as bases de dados dos quatro programas. Comparações entre os programas, dois a dois, foram efetuadas com o auxílio do teste de Mann-Whitney. A escolha de testes estatísticos não paramétricos justifica-se em razão de as variáveis em estudo não apresentarem distribuição normal. O nível de significância adotado foi de $5 \%$. Para as análises foi utilizado o programa Statistical Package for Social Sciences (SPSS) versão 10.0 . 
Tabela 1. Grupos alimentares e alimentos selecionados para a construção de 10 cardápios de 2200 kcal. Florianópolis (SC), 2006.

\begin{tabular}{|c|c|c|c|c|}
\hline Grupos alimentares & & Alimentos & selecionados & \\
\hline Frutas & $\begin{array}{l}\text { Abacaxi } \\
\text { Banana nanica } \\
\text { Cereja fresca } \\
\text { Goiaba } \\
\text { Jaca }\end{array}$ & $\begin{array}{l}\text { Pêssego } \\
\text { Kiwi } \\
\text { Laranja } \\
\text { Maçã } \\
\text { Manga }\end{array}$ & $\begin{array}{l}\text { Melancia } \\
\text { Melão } \\
\text { Morango } \\
\text { Nectarina } \\
\text { Pêra }\end{array}$ & $\begin{array}{l}\text { Mamão papaia } \\
\text { Suco de abacaxi } \\
\text { Suco de laranja } \\
\text { Uva Itália } \\
\text { Uva rosada fresca }\end{array}$ \\
\hline Hortaliças & $\begin{array}{l}\text { Abóbora cozida } \\
\text { Abobrinha cozida } \\
\text { Agrião cru } \\
\text { Alface } \\
\text { Berinjela cozida } \\
\text { Beterraba crua }\end{array}$ & $\begin{array}{l}\text { Salsão } \\
\text { Tomate } \\
\text { Pepino cru } \\
\text { Pepino enlatado } \\
\text { Chuchu cozido } \\
\text { Ervilha enlatada }\end{array}$ & $\begin{array}{l}\text { Escarola crua } \\
\text { Espinafre cozido } \\
\text { Palmito enlatado } \\
\text { Rabanete cru } \\
\text { Vagem cozida } \\
\text { Brócolis cozido }\end{array}$ & $\begin{array}{l}\text { Broto de feijão cozido } \\
\text { Repolho cozido } \\
\text { Repolho roxo cru } \\
\text { Pimentão verde } \\
\text { Couve flor cozida } \\
\text { Cenoura cozida }\end{array}$ \\
\hline Óleos e gorduras & $\begin{array}{l}\text { Banha de porco } \\
\text { Óleo soja }\end{array}$ & $\begin{array}{l}\text { Manteiga } \\
\text { Margarina }\end{array}$ & $\begin{array}{l}\text { Óleo girassol } \\
\text { Óleo milho }\end{array}$ & Azeite de oliva \\
\hline Carnes e ovos & $\begin{array}{l}\text { Camarão cozido } \\
\text { Carne de boi moída }\end{array}$ & Lingüiça de carne suína & Ovo de galinha frito & $\begin{array}{l}\text { Peito de frango sem } \\
\text { pele assado }\end{array}$ \\
\hline Leguminosas & $\begin{array}{l}\text { Feijão preto cozido } \\
\text { Soja cozida }\end{array}$ & $\begin{array}{l}\text { Lentilha cozida } \\
\text { Ervilha cozida }\end{array}$ & Feijão branco cozido & Grão de bico cozido \\
\hline Pães, cereais, raízes e tubérculos & $\begin{array}{l}\text { Arroz integral cozido } \\
\text { Batata doce cozida } \\
\text { Milho verde enlatado } \\
\text { Cereal matinal tipo flo- } \\
\text { cos de milho com açúcar } \\
\text { Biscoito recheado } \\
\text { chocolate }\end{array}$ & $\begin{array}{l}\text { Farinha milho } \\
\text { Inhame cozido } \\
\text { Mandioca } \\
\text { Pão caseiro } \\
\text { Pão francês } \\
\text { Biscoito aveia }\end{array}$ & $\begin{array}{l}\text { Macarrão cozido } \\
\text { Purê de batata } \\
\text { Pão cachorro quente } \\
\text { Batata frita } \\
\text { Batata cozida }\end{array}$ & $\begin{array}{l}\text { Arroz branco cozido } \\
\text { Biscoito cream cracker } \\
\text { Biscoito Wafer chocolate } \\
\text { Bolo de chocolate sem } \\
\text { recheio sem cobertura } \\
\text { Farinha de mandioca }\end{array}$ \\
\hline Produtos lácteos & $\begin{array}{l}\text { Leite em pó integral } \\
\text { Queijo minas fresco } \\
\text { Pudim de leite } \\
\text { Leite desnatado }\end{array}$ & $\begin{array}{l}\text { Requeijão } \\
\text { Ricota } \\
\text { Queijo prato } \\
\text { Queijo parmesão }\end{array}$ & $\begin{array}{l}\text { Queijo mussarela } \\
\text { Queijo provolone } \\
\text { Molho branco }\end{array}$ & $\begin{array}{l}\text { logurte frutas magro } \\
\text { logurte natural integral } \\
\text { Leite de vaca integral }\end{array}$ \\
\hline Doces & Açúcar refinado & Açúcar mascavo & Mel de abelha & Geléia de frutas \\
\hline
\end{tabular}

\section{RES U LTA D OS}

A Tabela 2 apresenta as características gerais dos programas analisados. Constatou-se uma ampla variação do número de alimentos (934 no programa NutWin a 3815 no programa DietPro) e de nutrientes (28 no programa Virtual Nutri a 144 no programa DietPro) disponíveis nos programas. Os quatro programas analisados não apresentaram o álcool na distribuição percentual de energia e não permitiam a escolha de horários e número de refeições ilimitadas (por exemplo: mais de um lanche à tarde ou beliscos) na elaboração dos planos alimentares ou na avaliação do consumo alimentar (dados não apresentados na tabela).
Os programas NutWin e DietWin apresentam os valores ausentes dos nutrientes não disponíveis para determinado alimento como espaços em branco ( ), enquanto o Virtual Nutri atribui valor zero (0) a tais ausências. Por outro lado, no DietPro, os nutrientes não disponíveis para certos alimentos não são apresentados como parte de sua composição nutricional (dados não apresentados na tabela).

A Tabela 3 apresenta a mediana dos nutrientes avaliados nos quatro programas. Como o programa Virtual Nutri não analisa a gordura saturada, monoinsaturada e poliinsaturada, esses nutrientes não foram comparados por testes estatísticos com os demais programas. Diferenças significantes entre os quatro programas foram 
encontradas para os valores de fibra, ferro e sódio. O programa DietWin apresentou o maior valor desses três nutrientes enquanto que o Virtual Nutri apresentou o menor valor de fibra e o DietPro os menores valores de ferro e sódio. Entre os programas Nutwin, DietWin e DietPro também foi observada diferença significante para os valores de gordura monoinsaturada, sendo o menor valor encontrado no DietPro e o maior no NutWin.

Na comparação entre os programas dois a dois foram observadas diferenças significantes para os valores de gordura saturada, gordura monoinsaturada, gordura poliinsaturada, fibra, ferro e sódio. Para as diferenças estatisticamente significantes destaca-se que o programa DietPro apresentou menores valores de gordura saturada, quando comparado ao DietWin; menores valores de gordura monoinsaturada e poliinsaturada, quando comparado ao NutWin, e menores valores de ferro e sódio quando comparado aos três demais programas. Não foram encontradas diferenças estatisticamente significantes para nenhum dos nutrientes analisados, quando comparados os programas NutWin e DietWin. Em relação à fibra, o programa Virtual Nutri apresentou valores significantemente menores, quando comparado a todos os outros programas.

Tabela 2. Características operacionais dos programas de análise de consumo alimentar. Florianópolis (SC), 2006.

\begin{tabular}{|c|c|c|c|c|}
\hline Características & NutWin ${ }^{13}$ & $\operatorname{DietWin}^{14}$ & DietPro $^{15}$ & Virtual Nutri ${ }^{16}$ \\
\hline Número de alimentos na base de dados & 934 & 1600 & 3815 & 1317 \\
\hline Número de nutrientes disponíveis & 77 & 112 & 144 & 28 \\
\hline Principais fontes & $a, f, l$ & $a, e, g, h, j, l$ & $a, g, h, i, j$ & $a, b, c, d, e$ \\
\hline Exporta dados & Sim & Sim & Sim & Não \\
\hline Compara dados com padrões dietéticos & Sim & Sim & Sim & Sim \\
\hline Armazena arquivos ou pastas & Sim & Sim & Sim & Sim \\
\hline Imprime relatórios & Sim & Sim & Sim & Sim \\
\hline Utiliza porção em medida caseira & Sim & Sim & Sim & Sim \\
\hline
\end{tabular}

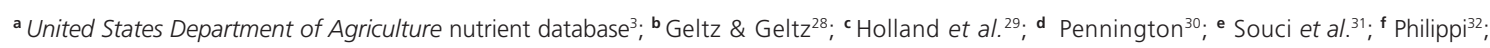
g Mendez et al. ${ }^{25}$; $\mathbf{h}$ Instituto Brasileiro de Geografia e Estatística24; i Pinheiro et al. ${ }^{33}$; j Lajolo \& Menezes ${ }^{34 ;}$, $^{2}$ Franco $^{22}$.

Tabela 3. Valores medianos de energia (Kcal) e nutrientes (gramas e miligramas) obtidos a partir de 10 cardápios analisados por quatro programas de análise de consumo alimentar. Florianópolis (SC), 2006.

\begin{tabular}{|c|c|c|c|c|c|}
\hline Variáveis & NutWin ${ }^{13}$ & DietWin $^{14}$ & DietPro $^{15}$ & Virtual Nutri ${ }^{16}$ & $p^{1}$ \\
\hline Energia (Kcal) & 2372,9 & 2273,6 & 2238,6 & 2356,1 & 0,54 \\
\hline Proteína (g) & 95,1 & 88,2 & 96,0 & 98,8 & 1,00 \\
\hline Carboidrato (g) & 365,6 & 331,8 & 338,0 & 350,8 & 0,16 \\
\hline Gordura total (g) & 57,1 & 66,5 & 68,8 & 65,7 & 1,00 \\
\hline Gordura saturada (g) & 20,6 & 21,5 & $14,7^{\mathrm{a}}$ & ND & 0,06 \\
\hline Gordura monoinsaturada (g) & 20,3 & 18,1 & $13,7^{\mathbf{b}}$ & ND & 0,03 \\
\hline Gordura poliinsaturada (g) & 12,2 & 8,0 & $7,6^{\mathbf{b}}$ & ND & 0,12 \\
\hline Colesterol (mg) & 199,7 & 212,8 & 182,9 & 184,5 & 0,65 \\
\hline Fibra (g) & 30,0 & 31,2 & $28,0^{c}$ & $22,3^{\text {d e }}$ & 0,01 \\
\hline Ferro (mg) & 18,2 & 18,8 & $9,0^{a b c}$ & 15,5 & $<0,01$ \\
\hline Cálcio (mg) & 1029,2 & 1041,2 & 908,8 & 964,3 & 0,46 \\
\hline Sódio (mg) & 1490,2 & 1953,5 & $611,4^{a b c}$ & 1528,5 & $<0,01$ \\
\hline
\end{tabular}

ND: não disponível.

1. Teste de Kruskall Wallis.

Teste Mann-Whitney: ${ }^{\text {a: }}$ diferença significativa entre DietWin e DietPro; ${ }^{\mathbf{b}}$ : diferença significativa entre NutWin e DietPro; ${ }^{c}$ : diferença significativa entre Virtual Nutri e DietPro; d: diferença significativa entre DietWin e Virtual Nutri; e: diferença significativa entre NutWin e Virtual Nutri. 


\section{DISCUSSÃO}

O tipo de comparação realizada neste estudo apresenta algumas limitações.

Com a finalidade de fundamentar a elaboração de 10 cardápios com base em princípios nutricionais, foi utilizada como referência a dieta de 2 200Kcal, estabelecida pelo guia da Pirâmide Alimentar Adaptada para a População Brasileira ${ }^{20}$. O mesmo procedimento pode ser aplicado tendo-se como referência a dieta de 2000 kcal recomendada no Guia Alimentar da População Brasileira ${ }^{23}$

Os 10 cardápios elaborados foram constituídos de alimentos básicos, de consumo popular, não sendo incluídas preparações elaboradas e produtos com marcas comerciais. Variações de maior ou menor magnitude poderiam ocorrer com a inclusão de tais alimentos. Além disso, a análise foi limitada ao valor energético e de 11 nutrientes disponíveis nas bases de dados. Não foram analisadas as vitaminas, os aminoácidos e os ácidos graxos (por exemplo, ácido linoléico e linolênico), pois não estavam disponíveis na maioria das tabelas de composição de alimentos, utilizados como fontes para compor a base de dados dos programas analisados ${ }^{22,24,25}$. Outra limitação referese à ausência de um padrão de referência para comparação dos valores de nutrientes obtidos nos quatro programas, fato que impossibilita a inferência de hipóteses sobre superestimativas e subestimativas, limitando a interpretação dos resultados. Dados provenientes da análise química de nutrientes são considerados padrão-ouro para comparação de bases de dados de programas, uma vez que oferecem uma melhor aproximação da composição nutricional dos alimentos ${ }^{7,12}$. Os valores de energia e de macronutrientes de alimentos analisados no laboratório podem diferir significativamente dos valores fornecidos por tabelas de composição e programas, os quais, dependendo do programa utilizado na comparação, podem sub ou superestimar os dados em relação às análises químicas ${ }^{12}$.

Os resultados obtidos neste estudo mostraram que os programas de análise de nutrientes variaram em relação às características gerais e aos valores de nutrientes de suas bases de dados. Relativamente às características gerais dos quatro programas analisados, evidenciaram-se diferenças na disponibilidade de alimentos, nos nutrientes e no modo de apresentação dos valores ausentes. Por outro lado, os programas desempenham funções semelhantes quanto à capacidade de comparar dados com padrões dietéticos, imprimir relatórios, armazenar arquivos ou pastas e utilizar tamanhos de porções em medidas caseiras, características importantes para a escolha de um programa de análise de nutrientes ${ }^{4}$.

Dentre as limitações operacionais apresentadas pelos programas citam-se a indisponibilidade de inclusão de outras refeições ou lanches e dos horários de realização desses eventos alimentares. Este fato dificulta, por exemplo, a elaboração de dietas fracionadas para pacientes que necessitam de uma prescrição dietoterápica especializada, a avaliação do consumo alimentar dos pequenos lanches ou beliscos e a análise dos ritmos circadianos de consumo alimentar ${ }^{26}$. Além disso, nenhum dos programas analisados considera o álcool na distribuição percentual da quantidade de energia total, podendo conduzir a interpretações equivocadas relativamente às proporções de energia proveniente de proteínas, carboidratos e gorduras, no caso do consumo de bebidas alcoólicas.

As análises realizadas não permitiram isolar e quantificar os fatores responsáveis pelas diferenças de valores de composição de nutrientes encontrados, devido às várias fontes de dados utilizadas pelos programas. Diversos fatores podem ter contribuído para as variações de composição observadas neste estudo. Primeiro, a diferença do número de alimentos de cada programa conduziu a graus variados de substituição de alimentos ou de suas formas de preparo, quando os cardápios foram digitados em cada programa. Além disso, as múltiplas formas de descrição dos alimentos entre os programas podem ter contribuído para as diferenças encontradas nas quantidades dos nutrientes estudados. Por 
exemplo, foram observadas diferenças na possibilidade de selecionar modos de preparo: alimento in natura (cru), alimentos submetidos a processos de cocção (assado, cozido, grelhado, frito, ensopado) e alimentos preparados com ou sem a adição de sal e óleo. Assim, a descrição diferenciada de alimentos semelhantes exerceu influência nos resultados observados relativamente aos valores de sódio e dos tipos de gorduras, os quais apresentam relação direta com os modos de preparo dos alimentos. Segundo, um mesmo alimento apresentou valores diferentes de nutrientes entre os quatro programas, como, por exemplo, o leite de vaca integral. As informações nutricionais da maioria das tabelas de composição de alimentos, utilizadas pelos diferentes programas, são provenientes de fontes de dados secundárias, aumentando a possibilidade de variação entre as fontes, seja por equívocos provenientes da tradução dos alimentos e seus nutrientes, seja por erro na digitação de suas quantidades. Por outro lado, as fontes das bases de dados sofrem influência dos métodos de análise química usados para quantificar o conteúdo de nutrientes dos alimentos, sendo, pois, difícil, presumir de onde se originam as diferenças entre os programas, quando estes se baseiam em diferentes fontes de dados. Além disso, como as bases de dados dos programas são originadas de tabelas provenientes de várias regiões do mundo, as diferenças observadas podem refletir as variações da própria composição dos alimentos: por exemplo, a quantidade de gordura do leite varia de acordo com a raça e a alimentação do animal, o teor de nutrientes dos grãos, das frutas e dos vegetais varia de acordo com as técnicas agrícolas e as condições climáticas tais como, chuva ou seca ${ }^{11}$. Finalmente, as variações observadas entre as bases de dados de nutrientes dos quatro programas podem, ainda, ser atribuídas a diferenças no número de nutrientes com valores ausentes e no modo de apresentação desses dados.

Ressalte-se que essa indefinição de resultados decorrente de múltiplas influências parece ser a tônica das pesquisas nesta área. A comparação destes resultados com outros estudos sobre composição de consumo alimentar e ingestão de nutrientes gerados por diferentes programas é limitada pelas diferenças no delineamento da pesquisa, pelos tipos de alimentos analisados, pelos padrões de referência e pelos testes estatísticos utilizados nas análises de dados.

No presente estudo não foram encontradas diferenças significantes entre os programas para os valores de energia e de macronutrientes, contudo os valores de fibra, ferro, sódio e gordura monoinsaturada diferiram entre eles. Na revisão da literatura pertinente a este tema, foram constatadas diferenças significantes para alguns nutrientes sempre que os pesquisadores utilizaram diferentes programas para a análise das mesmas dietas. Por exemplo, Shanklin et al. ${ }^{9}$ encontraram diferenças significantes para os valores de energia, proteína, gordura total, niacina, riboflavina, vitamina C, sódio, potássio, fósforo e ácido linoléico, na comparação de dados de ingestão de nutrientes calculados a partir de recordatórios de 24 horas (R24h), utilizando a base de dados de nutrientes de dois programas. Eck et al. ${ }^{5}$ verificaram diferenças significantes para os valores de energia, proteína, gordura total, carboidrato e ferro obtidos em 60 R24h, ao compararem os dados obtidos com a utilização de 4 bases de dados de nutrientes. Por outro lado, Taylor et al. ${ }^{10}$ ao compararem os valores de nutrientes obtidos em 24 registros alimentares, com a utilização de 3 programas, não observaram diferenças para os valores de energia e macronutrientes, mas encontraram diferenças significantes para os valores de ferro, vitamina $A$, tiamina, riboflavina, vitamina $C$, ácido fólico, vitamina $B_{6}$, vitamina $D$ e zinco.

Outros estudos ${ }^{6,11}$ conduziram comparações de bases de dados de nutrientes de programas de países diferentes. Hakala et al. ${ }^{6}$ compararam dados de consumo alimentar de 20 homens adultos entre programas de computador sueco e finlandês, observando diferenças significantes para os valores de proteína, gordura monoinsaturada, gordura poliinsaturada, tiamina, vitamina $B_{6}$, folato, zinco, cálcio, magnésio, potássio, selênio, ferro e sódio dentre 29 nutrientes analisados. Vaask 
et al. ${ }^{11}$ compararam a base de dados de nutrientes entre um programa utilizado na Estônia e um outro utilizado na Lituânia e Letônia, a partir do conteúdo de nutrientes de 15 alimentos comumente consumidos nos países bálticos e de 32 R24h. Segundo os autores, os programas diferiram nos valores de energia, carboidrato, proteína, lipídeo, cálcio, vitamina $C$ e ferro para os 15 alimentos selecionados. Nos R24h foram encontradas diferenças estatisticamente significantes para os valores de energia, gordura total, carboidrato e cálcio.

Lee et al. ${ }^{3}$ compararam os valores dos nutrientes de três registros alimentares calculados por meio de oito programas de análise de consumo alimentar utilizando a base de dados de nutrientes do United States Departament of Agriculture (USDA) como tabela de referência. Os programas produziram resultados semelhantes à base de dados de referência para os valores de energia, proteína, gordura total e carboidratos. Variaram, contudo, no que se referiu a fibras, ácidos graxos, colesterol e $\alpha$ tocoferol.

No estudo de McCullough et al. ${ }^{7}$ os dados de nutrientes obtidos com o cálculo de 36 cardápios, por meio de 4 programas, foram comparados com os resultados da análise química laboratorial desses cardápios. Diferenças significantes foram relatadas para os ácidos graxos saturados, monoinsaturados e poliinsaturados, potássio, magnésio e ferro.

A incorporação dos dados da Tabela Brasileira de Composição de Alimentos (TACO) ${ }^{27}$ em programas computadorizados de planejamento e avaliação de consumo alimentar permitirá novas comparações com outras bases de dados de nutrientes.

\section{O N CLUS Ã O}

Os resultados desta pesquisa mostraram que na avaliação de energia e nutrientes de 10 cardápios calculados com diferentes programas de análise de consumo alimentar, valores dife- rentes foram encontrados para gordura monoinsaturada, fibras, ferro e sódio. Estes resultados indicam a possibilidade de comparar os dados de energia e de macronutrientes obtidos em estudos de consumo alimentar, evidenciando, no entanto, necessidade de cautela no que concerne aos demais nutrientes. Tais resultados não permitem, ainda, inferências quanto à qualidade das bases de dados e nem indicam a superioridade de quaisquer desses programas que justifique sua indicação preferencial na prática do profissional de nutrição ou do pesquisador do consumo alimentar

Sugere-se a utilização de dieta padrão de órgãos oficiais, como o Guia Alimentar para a População Brasileira do Ministério da Saúde, em futuras pesquisas de comparação de valores de energia e nutrientes obtidos com diferentes programas computadorizados, além de pesquisas com tais programas conduzidas com dados de consumo alimentar provenientes de registros e recordatórios alimentares. Estudos dessa natureza poderão contribuir para aprimorar o delineamento dos programas computadorizados.

\section{A GRADECIMENTO}

A Coordenação de Aperfeiçoamento de Pessoal de Nível Superior, pela concessão da bolsa de mestrado a F.G.K. Vieira.

\section{OLA B OR A Ç Ã O}

F.G.K. VIEIRA, P.F. Di PIETRO, L.C. FEIO, M.A.A. ASSIS e F.A.G. VASCONCELOS participaram do delineamento do estudo, análise e interpretação dos dados. M.A. PERES participou da análise estatística e interpretação dos dados. Todos os autores participaram da redação do manuscrito.

\section{REFERÊ N CIAS}

1. Achenson KJ, Campbell IT, Edholm OG, Miller DS, Stock MJ. The measurement of food and energy intake in man: an evaluation of some techniques. Am J Clin Nutr. 1980; 33(5):1147-54. 
2. Cuppari L, Anção MS. Uso de programas computadorizados na avaliação dietética. In: Fisberg RM, Slater E, Marchioni DML, Martini LA, editores. Inquéritos alimentares: métodos e bases científicos. São Paulo: Manole; 2005. v.1.

3. Lee RD, Nieman DC, Rainwater M. Comparison of eight microcomputer dietary analysis programs with the USDA nutrient data base for standard reference. J Am Diet Assoc. 1995; 95(8):858-67.

4. Vozenilek GP. Choosing the best nutrient analysis software for your needs. J Am Diet Assoc. 1999: 99(11):1356-7.

5. Eck LH, Klesges RC, Hanson CL, Baranowski T, Henske J. A comparison of four commonly used nutrient database programs. J Am Diet Assoc. 1988; 88(5):602-4

6. Hakala P, Knuts LR, Vuorinen A, Hammar N, Becker W. Comparison of nutrient intake data calculated on the basis of two different databases. Results and experiences from a Swedish-Finnish study. Eur J Clin Nutr. 2003; 57(9):1035-44.

7. McCullough ML, Karanja NM, Lin PH, Obarzanek E, Phillips KM, Laws RL, et al. Comparison of 4 nutrient databases with chemical composition data from the dietary approaches to stop hypertension trial. J Am Diet Assoc. 1999; 99(S8):S45-53.

8. Nieman DC, Butterworth DE, Nieman CN, Lee KE, Lee RD. Comparison of six microcomputer dietary analysis systems with the USDA nutrient database for standard reference. J Am Diet Assoc. 1992; 92(1):48-56

9. Shanklin D, Endres JM, Sawicki M. A comparative study of two nutrient databases. J Am Diet Assoc. 1985; 85(3):308-13.

10. Taylor ML, Kozlowski BW, Baer MT. Energy and nutrient values from different computerized databases. J Am Diet Assoc. 1985; 85(9):1136-8.

11. Vaask S, Pomerleau J, Pudule I, Grinberga D, Abaravicius A, Robertson A, et al. Comparison of the micro-nutrica nutritional analysis program and the Russian food composition database using data from the Baltic nutrition surveys. Eur J Clin Nutr. 2004; 58(4):573-9.

12. Ribeiro P, Morais TB, Colugnati FAB, Sigulem DM. Tabelas de composição química de alimentos: análise comparativa com resultados laboratoriais. Rev Saúde Pública. 2003; 37(2):216-25.

13. Anção MS, Cuppari L, Tudisco ES, Draibe SA, Sigulem DM. Sistema de Apoio à Nutrição. NutWin [programa de computador]. Versão 2.5. São Paulo: Centro de Informática em Saúde, Universidade Federal de São Paulo/Escola Paulista de Medicina; 2002.
14. Software de avaliação nutricional. DietWin clínico [programa de computador]. Versão 3.0. Porto Alegre: Brubins Comércio de Alimentos e Supergelados; 2002.

15. Bressan J, Esteves E. Sistema de suporte de avaliação nutricional e avaliação de dietas. DietPro [programa de computador]. Versão 4.0. Minas Gerais: Agromídia software; 2001.

16. Philippi ST, Szarfac SC, Latterza AR. Virtual Nutri [programa de computador]. Versão 1.0 for Windows. São Paulo: Departamento de Nutrição da Faculdade de Saúde Pública da Universidade de São Paulo; 1996

17. Castro TG, Novaes, JF, Silva, MR, Costa, NMB, Franceschini SCC, Tinoco ALA, et al. Caracterização do consumo alimentar, ambiente sócio-econômico e estado nutricional de pré-escolares de creches municipais. Rev Nutr. 2005; 18(3):321-30.

18. Fisberg RM, Morimoto JM, Marchioni DML, Slater B. Using dietary reference intake to evaluate energy and macronutrient intake among young women Nutr Res. 2006; 26(4):151-3.

19. Gomes AIS, Ribeiro BG, Soares EA. Caracterização nutricional de jogadores de elite de futebol de amputados. Rev Bras Med Esporte. 2005; 11(1): 11-6.

20. Philippi ST, Latterza AR, Cruz ATR, Ribeiro LC. Pirâmide alimentar adaptada: guia para escolha dos alimentos. Rev Nutr. 1999; 12(1):65-80.

21. Brasil. Ministério da Saúde. Agência Nacional de Vigilância Sanitária. Rotulagem nutricional obrigatória: manual de orientações às indústrias de alimentos. 2a. Versão. Brasília; 2005 [acesso 2007 16 fev]. Disponível em: <www.anvisa.gov.br/rotulo/ manual_industria>.

22. Franco G. Tabela de composição química dos alimentos. 9a. ed. Rio de Janeiro: Atheneu; 1992.

23. BRASIL. Ministério da Saúde. Secretaria de Atenção à Saúde Departamento de Atenção Básica. Coordenação-Geral da Política de Alimentação e Nutrição. Guia alimentar para a população brasileira: promovendo a alimentação saudável. Brasília: Ministério da Saúde; 2006.

24. Instituto Brasileiro de Geografia e Estatística. Estudo Nacional de Despesa Familiar (ENDEF). Tabelas de composição de alimentos. 5a. ed. Rio de Janeiro: IBGE; 1999.

25. Mendez MHM, Derivi SCN, Rodrigues MCR, Fernandes ML. Tabela de composição de alimentos: amiláceos, cereais e derivados, frutas, hortaliças, leguminosas, nozes e oleaginosas. Rio de Janeiro: Eduff; 1995.

26. Gauche H, Calvo MCM, Assis MAA. Ritmos circadianos de consumo alimentar nos lanches e 
refeições de adultos: aplicação do semanário alimentar. Rev Nutr. 2006; 19(2): 177-85.

27. Universidade Estadual de Campinas. Tabela brasileira de composição de alimentos - TACO, versão 2. Campinas: Nepa-Unicamp; 2006.

28. Geltz R, Geltz B. Food processor: nutrition and fitness software. Salem: Esha Research; 1984.

29. Holland B, Welch AA, Unwin ID, Buss DH, Paul AA, Southgate $D$, et al. The composition of foods. 5th ed. London: The Royal Society of Chemistry and Ministry of Agriculture; 1991.

30. Pennington JAT. Food values of portion commonly used. 15th ed. New York: Perennial Library; 1989.

31. Souci SW, Farchman W, Kraut K. Food composition and nutrition tables 89/90. 4th ed. Stutgart:
Deutsche Forschungsantalt fur Lebensmittelchemie; 1989.

32. Philippi ST. Tabela de composição de alimentos: suporte para decisão nutricional. 2a. ed. São Paulo: Coronário; 2002.

33. Pinheiro ABV, Lacerda EMA, Benzecry EH, Gomes MCS, Costa VM. Tabela para avaliação de consumo alimentar em medidas caseiras. Rio de Janeiro: Produção Independente; 1996.

34. Lajolo FM, Menezes EW. Tabela brasileira de composição de alimentos. Projeto integrado de composição de alimentos. São Paulo: USP; 1998.

Recebido em: 27/4/2007

Versão final reapresentada em: 25/9/2008 Aprovado em: 5/11/2008 